\title{
3D Vector Directional Filters to Process Video Sequences
}

\author{
Volodymyr Ponomaryov ${ }^{1}$, Francisco Gallegos-Funes ${ }^{2}$, Alberto Rosales-Silva ${ }^{1}$, \\ and Igor Loboda ${ }^{1}$ \\ ${ }^{1}$ National Polytechnic Institute of Mexico, \\ Mechanical and Electrical Engineering Higher School \\ ESIME-Culhuacan; Av. Santa Ana 1000, Col. San Francisco Culhuacan, \\ 04430, Mexico D.F., Mexico \\ vponomar@ipn.mx \\ ${ }^{2}$ ESIME-Zacatenco; Av. IPN s/n, U.P.A.L.M. Col. Lindavista, \\ 07738, Mexico D.F., Mexico \\ fgallegosf@ipn.mx
}

\begin{abstract}
We present novel algorithms to suppress impulsive noise in video color sequences. They use order statistics, directional and adaptive processing techniques. Extensive simulation results in known reference video color sequences have demonstrated that the proposed filters consistently outperform other filters by balancing the tradeoff between noise suppression, detail preservation, and chromaticity characteristics. The criteria used to compare the performance or various filters were the PSNR, MAE, and NCD.
\end{abstract}

Keywords: Adaptive and directional processing, Order statistics, 3D Video color sequences.

\section{Introduction}

Several filters have demonstrated good ability to remove impulsive noise, preserve fine details, and provide chromatic properties in multichannel processing applications [1-3]. One of the useful and promising approaches being proposed was the multichannel signal processing based on vector processing [1-3]. In this case the correlation in chromacity that exists between the channels is employed. The value of each a $2 \mathrm{D}$ pixel is represented by a $3 \mathrm{D}$ (three dimensional) vector, so, the color image is translated into a set of vectors with the directions and lengths that are related to the chromatic properties of the pixels. Nonlinear filtering techniques apply the robust order statistics theory that is the basis for design of the different novel approaches in digital multichannel processing [4-8].

In this paper we introduce novel methods to process 3D multichannel images that are based on order statistics and vectorial processing techniques [1,2]. We propose the Video Adaptive Vector Directional (VAVDF) filter. This filter can use in its filtering scheme the median or $\alpha$-trimmed mean filters to obtain the Video Adaptive Vector Directional Median (VAVDMF) filter and the Video Adaptive Vector Directional $\alpha$-trimmed mean (VAVDATMF) filter, respectively. We also proposed the Video Vector Directional K-Nearest Neighbour (VVDKNNF) filter with the 
estimation of vector median filter in its filtering scheme to obtain the VVDKNNVMF filter.

Finally, we present some adapted versions of $2 \mathrm{D}$ filters that can process $3 \mathrm{D}$ video colour sequences. The filters used during the implementations were: the median filter to obtain the Video Median filter, the Vector $\alpha$-trimmed mean (VATM) filter to realize the Video Alpha Trimmed Mean filter, the K-Nearest Neighbour filter that is adapted to implement the Video KNNF, and the Vector Median Filter to present the Video Vector Median (VVMF) Filter.

\section{Proposed Methods}

We denote an image pixel as $I(x, y, t)$, where $(x, y)$ and $t$ indicate the spatial and temporal location in the video sequence or, maybe third spatial coordinate in the case of $3 \mathrm{D}$ image, respectively. The $3 \mathrm{D}$ window (cube) contains the pixels $\left(x^{\prime}, y^{\prime}, t^{\prime}\right)$ where $\left|x-x^{\prime}\right|<3,\left|y-y^{\prime}\right|<3$ and $\left|t-t^{\prime}\right|<3$ [7]. The window is used to compute the filtered value $I^{\prime}(x, y, t)$.

\subsection{Video Generalized Vector Directional (VGVDF) Filter}

Vector directional processing is one of the most important techniques implemented recently $[4,7,8]$. The vector processing separates the signal vector processing in directional processing and magnitude processing. The pixels are denoted as vectors in $m$-dimensional spaces, in the case of color images, $m=3$. The vectors have to be ordered in certain way, in the majority of cases they are ordered as follows:

$$
\sum_{i=1}^{n} A\left(x_{B D}, x_{i}\right) \leq \sum_{i=1}^{n} A\left(x_{j}, x_{i}\right), \forall j=1,2, \ldots, n,
$$

where $A\left(x_{j}, x_{i}\right)$ is the angle among vectors $x_{i}$ and $x_{j}$, and $x_{B D}$ satisfies to minimum deviation value among vectors. So, $x_{B D}$ gives the output of known filter, the Basic Vector Directional Filter (BVDF). The Generalized Vector Directional Filter (GVDF) is selected from a set of vectors, which present the minimum deviation with respect to other vectors:

$$
\begin{gathered}
x^{(1)}=\operatorname{BVDF}\left[x_{1}, x_{2}, \ldots, x_{n}\right] \\
\left\{x^{(1)}, x^{(2)}, \ldots, x^{(k)}\right\}=G V D F\left[x_{1}, x_{2}, \ldots, x_{n}\right], \quad 1 \leq k \leq n \\
x_{0}=\mathfrak{I}\left\{x^{(1)}, x^{(2)}, \ldots, x^{(k)}\right\}=\mathfrak{I}\left\{G V D F\left[x_{1}, x_{2}, \ldots, x_{n}\right]\right\}
\end{gathered}
$$


According to (2)-(4) the GVDF produces set of vectors with typical directions and this set should be passed after through a magnitude processing filter $\mathfrak{I}$ to produce an only vector for each a pixel. The GVDF involves the $k$ parameter that needs to be specified for each particular implementation. This parameter can be selected in an adaptive and non adaptive way described in [1].

There are realized two operations using different windows to preserve image details and suppress noise effectively. We apply the following operations:

$$
x_{O}=\mathfrak{I}\left\{x^{(1)}, x^{(2)}, \ldots, x^{(K)}\right\}=\mathfrak{I}\left\{V G V D F\left[x_{1}, x_{2}, \ldots, x_{n}\right]\right\},
$$

where VGVDF is the Video Generalized Vector Directional Filter using to find the vectors with the minimum angle deviation from the vectors that are under processing $x_{2 j} \in W_{2}-W_{1}$ and $\alpha^{\prime}{ }_{2}$, which corresponds to $x_{2 j}$ and is defined as:

$$
\alpha_{2 j}^{\prime}=\sum_{i=1}^{n} A\left(x_{2 j}, x_{1 i}\right)
$$

During the next stage the vector $x_{2 j}$ is added to set $\left\{x_{1}^{(1)}, x_{1}^{(2)}, \ldots, x_{1}^{(k)}\right\}$ if the condition $\alpha_{2 j}^{\prime} \leq \alpha_{1}^{(k)}$ is satisfied. In this way, the external window contributes with vectors $x_{2 j}$ that diverges less than the last vector considered $x_{1}^{(k)}$ from $x_{1}$.

\subsection{Video $\alpha$-Trimmed Mean Filter}

The $\alpha$-trimmed mean filter is applicable to process color images [7]. In here, we adapt this filter to process $3 \mathrm{D}$ video color sequences.

All pixel values from the $3 \mathrm{D}$ window are ordered in one dimensional array according to order chosen:

$$
x_{L}=\left(x_{1}, x_{2}, \ldots, x_{N}\right)^{t} \text {, }
$$

where $N=27$ denotes the number of the pixels in the 3D sliding window.

Then, the pixels values in $x_{L}$ are ordered in ascending order to get a new 1D array,

$$
x_{\alpha}=\left(x_{(1)}, x_{(2)}, \ldots, x_{(N)}\right)^{t}
$$

where $x_{(1)}>x_{(2)}>\ldots>x_{(N)}$.

Finally, the filter output is calculated as follows:

$$
y=\frac{1}{\theta} \sum_{i=\left[\alpha^{*} N\right]+1}^{N-\left[\alpha^{*} N\right]} x_{(i)},
$$


where $\theta=N-2 *\left[\alpha^{*} N\right]$ and $[$.$] is the ceiling function, which rounds up to the$ nearest integer.

\subsection{Video Vector Directional K-Nearest Neighbour Vector Median (VVDKNNVMF) Filter}

The proposed Video KNN filter is based in the approach of ref. [7]. We sort the pixel values in $x_{L}$ according to their difference with $I(x, y, t)$ to get a new 1D array,

$$
x_{l}=\left(x_{(1)}, x_{(2)}, \ldots, x_{(N)}\right)^{t}
$$

where $x_{(1)}=I(x, y, t)$ is the central pixel en the sliding 3D window, $x_{(i)}, i=2,3, \ldots, N$ are the pixels that satisfy the condition $A\left(x_{(1)}, x_{(i)}\right) \leq A\left(x_{(1)}, x_{(j)}\right), j=i \ldots N$, and $A(\cdot)$ is the angle between the central pixel and the other N-1 pixels. So, the novel 1D array can be written as:

$$
x_{l}=\left(x_{(2)}, \ldots, x_{(N)}\right)^{t} .
$$

The set of the K-Nearest Neighbour vectors with respect to central pixel is obtained as,

$$
\left\{x^{(1)}, x^{(2)}, \ldots, x^{(K)}\right\}^{t}=\operatorname{VDKNNF}\left[x_{(2)}, \ldots, x_{(N)}\right]^{t}, 1 \leq K \leq(N-1) .
$$

The first $K$ terms of the ordered sequence $\left\{x^{(i)}\right\}^{t}$ constitute the output of VDKNN (Vector Directional $K$-Nearest Neighbour) filter. At the final stage the magnitude processing filter should be applied to obtain an only output vector for each a pixel. It is done using the Vector Median (VMF) Filter that defines the VVDKNNVMF to process 3D video sequences:

$$
V M F\left\{x^{(1)}, x^{(2)}, \ldots, x^{(K)}\right\}^{t}=x_{V M F} .
$$

\section{Experimental Results}

During the simulations the $176 \times 144$ video sequences "Miss America" and "Flowers" were corrupted with noise of different intensity. The noise was independently added in each a plane of the frame. The criteria applied to evaluate the performance of various filters were: the Pick Signal-to-Noise Relation (PSNR) used to characterize noise suppression level, the Mean Absolute Error (MAE), which characterizes a 
quality of edge and detail preservation, and the Normalized Color Difference (NCD) to qualify and quantify chromaticity preservation given by color perceptual error.

The proposed Video Generalized Vector Directional (VGVDF) Filter with the $\alpha$ trimmed mean and median filters as a magnitude ones, the Video Adaptive Vector Directional $\alpha$-trimmed mean (VAVDATM), the Video Adaptive Vector Directional median (VAVDMF) filters, the Video Vector Directional K-Nearest Neighbour (VVDKNNF) filter, and the VVDKNNVMF filter that have been introduced in this paper were compared with other filters proposed here.

We utilize the following filters as a comparative ones to evaluate the properties of noise suppression, detail preservation, and chromaticity preservation of the proposed filters: the median filter that was been adapted to 3D processing to obtain the Video MF, the Vector Median Filter that gives the Video Vector Median (VVMF) Filter, the Vector ATM filter improving the Video ATM, and the KNNF [1] in two variants in the $3 \times 3 \times 3$ window named as filter $\mathrm{KNNF}_{-} 1$, and in the CrossXCrossXCross window named as KNNF_2, These filters were adapted to realize 3D processing and to obtain the Video KNN Filter.

Table 1 presents the comparative filtering results in a frame of video sequences "Flowers" and "Miss America" obtained by different filters. From the performance results one can see that the proposed filters demonstrate the best results in the terms of criteria used.

Table 1. Comparative results in a frame of video sequences "Flowers" and "Miss America"

\begin{tabular}{|l|c|c|c|c|c|c|}
\hline \multirow{2}{*}{ Algorithm } & \multicolumn{3}{|c|}{ Flowers (15\%) } & \multicolumn{3}{c|}{ Miss America (20\%) } \\
\cline { 2 - 7 } & PSNR & MAE & NCD & PSNR & MAE & NCD \\
\hline VATM & 27,002 & 6,305 & 0,01260 & 34,585 & 2,534 & 0,00906 \\
\hline KNNF_1 & 26,144 & 7,386 & 0,01398 & 28,113 & 5,477 & 0,02021 \\
\hline KNNF_2 & 26,903 & 6,140 & 0,01150 & 27,644 & 5,144 & 0,01929 \\
\hline VAVMF & 25,384 & 6,996 & 0,01300 & 32,099 & 2,876 & 0,00989 \\
\hline Video-MF & 26,973 & 6,150 & 0,01241 & 34,571 & 2,454 & 0,00917 \\
\hline VVMF & 26,919 & 6,108 & 0,01235 & 34,446 & 2,413 & 0,00876 \\
\hline VAVDMF_1 & 26,722 & 5,871 & 0,01209 & 33,256 & 2,196 & 0,00836 \\
\hline VAVDMF_2 & 27,285 & 5,240 & 0,01059 & 35,237 & 1,719 & 0,00674 \\
\hline VAVDATM & 27,331 & 5,328 & 0,01068 & 35,25 & 1,765 & 0,00664 \\
\hline VVDKNNVMF & 26,176 & 6,932 & 0,01399 & 32,292 & 3,208 & 0,0113 \\
\hline
\end{tabular}

Figure 1 displays the filtered frames and error images in the case of $15 \%$ of impulsive noise contamination for video color sequence "Flowers". This figure clearly shows that the Video Adaptive Vector Directional Alpha-trimmed Mean (VAVDATM) filter can suppress noise corruption and provide detail preservation better than other filters.

Figure 2 presents the processed images in the case of $30 \%$ impulsive noise in a frame of video sequence "Miss America". The restored image found applying the proposed filters appears to have a very good subjective quality. 


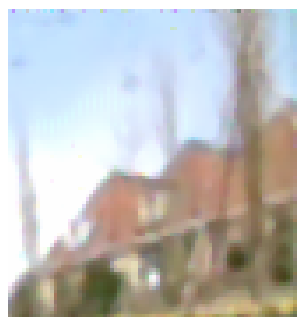

a)

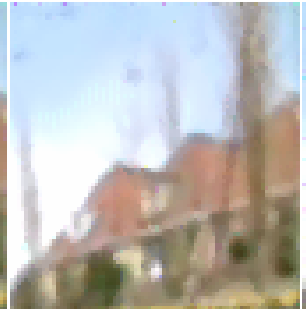

b)

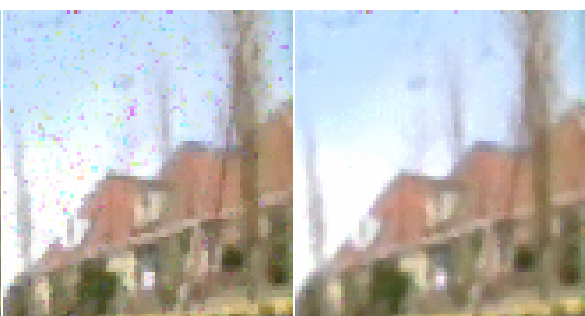

c)

d)

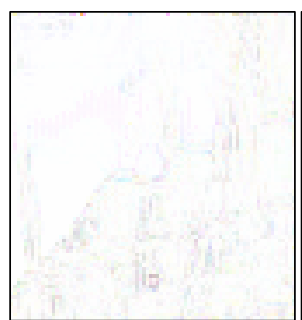

e)

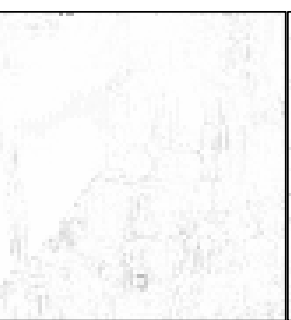

f)

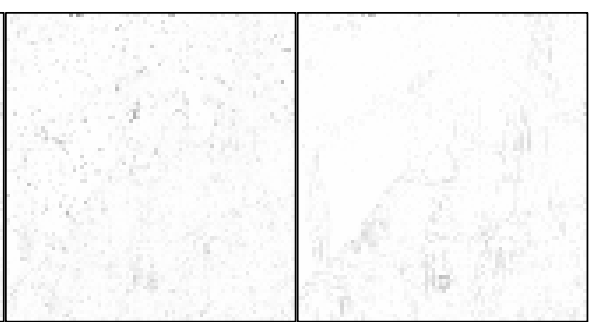

g)

h)

Fig. 1. Visual results in a frame of video sequence "Flowers", a) Restored frame applying VATM filter, b) Restored frame applying VVMF Filter, c) Restored frame applying KNNF_1 filter, d) Restored frame applying VAVDATM filter, e) Error image applying VATM filter, f) Error image applying VVMF Filter, g) Error image applying KNNF_1 filter, h) Error image applying VAVDATM filter

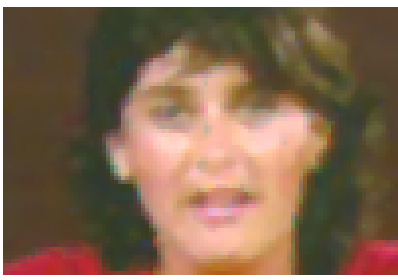

a)

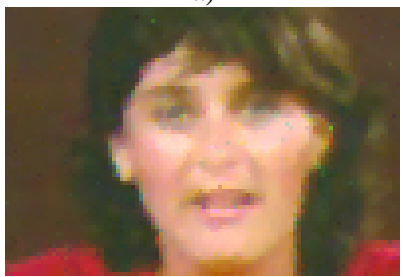

c)

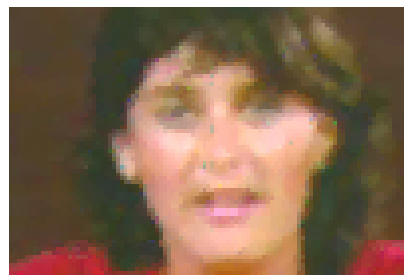

b)

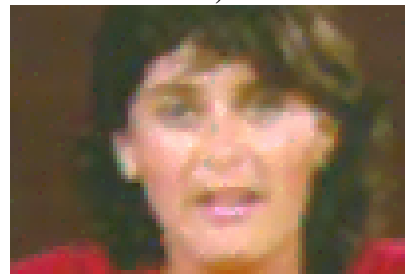

d)

Fig. 2. Filtered Images with $30 \%$ of impulsive noise in a frame of video sequence "Miss America": a) Restored frame applying VATM filter, b) Restored frame applying VVMF filter, c) Restored frame applying VMMKNN filter [6], and d) Restored image applying VAVDATM filter 


\section{Conclusions}

Proposed filtering methods have demonstrated their effectiveness in noise suppression, detail preservation and chromaticity characteristics. The novel VAVDATM and VAVDMF filters appear to demonstrate the best results in terms criteria used and visually in noise suppression and detail and color preservation. Several filters that work in 2D processing have been adapted in here to process 3D video information demonstrating good properties in terms of criteria PSNR, MAE, and NCD.

Acknowledgments. This work is supported by National Polytechnic Institute of Mexico and CONACyT (project 42790).

\section{References}

1. Trahanias P. E., Karakos D., Venetsanopoulos A. N.: Direccional Processing of Color Images: Theory and Experimental Results. IEEE Trans. on Image Process. Vol. 5 No. 6. (1996) 868-880.

2. Plataniotis K. N., Androutsos D., Vinayagamoorthy S., Venetsanopoulos A. N.: Color Image Processing Using Adaptive Multichannel Filters. IEEE Trans. on Image Process. Vol. 6 No. 7. (1997) 933-949.

3. Shkvarko YV.: Unifying regularization and Bayesian Estimation methods for enhanced imaging with remotely sensed data. IEEE Trans. On GSRS, Vol. 42 No. 5. (2004) 923-931.

4. Lukac R.: Adaptive Vector Median Filtering. Pattern Recognition Lett. Vol. 24. (2003) 1889-1899.

5. Gallegos-Funes F., Ponomaryov V., De-La-Rosa J.: ABST M-type K-nearest neighbor (ABSTM-KNN) for image denoising. IEICE Trans. on Funds. of Electronics Comms. and Computer. Science. Vol.E88A, No.3, (2005) 798-799.

6. Ponomaryov V. I., Gallegos-Funes F. J., Rosales-Silva A.: Real-Time Color Imaging Based on RM-Filters for Impulsive Noise Reduction. J. Imaging Science and Technology, Vol. 49, No. 3. (2005) 205-219.

7. Zlokolica V., Philips D., Van De Ville D.: A New Non-Linear Filter for Video Processing. IEEE Benelux Signal Processing Symposium, March 2002, Belgium

8. Koschan T., Abidi M.: A comparison of Median Filter Techniques for Noise Removal in Color Images. Proc. $7^{\text {th }}$ German Workshop on Color Image Processing, Vol. 34, No. 15, (2001) 69-79. 